\title{
A SURVEY OF PRODUCT-FORM QUEUEING NETWORKS WITH BLOCKING AND THEIR EQUIVALENCES
}

\author{
Simonetta BALSAMO * \\ and \\ Vittoria DE NITTO PERSONE' 。 \\ * Department of Computer Science, University of Pisa, Pisa, Italy \\ 'Department of Elect. Engineering, University of Roma Tor Vergata, Rome, Italy
}

\begin{abstract}
Queueing network models have been extensively used to represent and analyze resource sharing systems, such as production, communication and information systems. Queueing networks with blocking are used to represent systems with finite capacity resources and with resource constraints. Different blocking mechanisms have been defined and analyzed in the literature to represent distinct behaviors of real systems with limited resources. Exact product form solutions of queueing networks with blocking have been derived, under special constraints, for different blocking mechanisms. In this paper we present a survey of product form solutions of queueing networks with blocking and equivalence properties among different blocking network models. By using such equivalences we can extend product form solution to queueing network models with different blocking mechanisms. The equivalence properties include relationships between open and closed product form queueing networks with different blocking mechanisms.
\end{abstract}

Index terms - queueing networks, finite capacity queue, blocking, equivalence, performance analysis, product-form.

\footnotetext{
This work has been partially supported by CNR Project Research Funds 'Progetto Finalizzato Sistemi Informatici e Calcolo Parallelo' and by MURST Project Research Funds 'Performability hw/sw di sistemi distribuiti e paralleli'.
} 


\section{Introduction}

Queueing networks are used to represent and to analyze resource sharing systems, such as production, telecommunication and information systems, and they have proved to be a powerful tool for performance evaluation and prediction [23, 28]. Queueing networks have been analyzed and solved under different assumptions and constraints. Various classes of queueing networks have been shown to have product form solutions, and efficient algorithms to exactly evaluate their performance have been developed $[9,28]$. Most of the product form queueing network models require infinite capacity queues. Queueing network models with finite capacity queues have been introduced in order to represent real systems with finite capacity resources. In these models, when a queue reaches its maximum capacity, the flow of customers entering the service center is stopped, both from other service centers and from external sources in case of open networks. This phenomenon is referrred to as blocking.

Queueing networks with blocking can be applied to telecommunication systems and computer systems with limited shared resources, such as interconnecting links or store and forward buffers, as well as in production systems with the finite storage buffers.

Different blocking mechanisms representing distinct behaviors of real systems with limited resources have been defined and analyzed in the literature. Some of the reported blocking mechanisms have been recently named and classified by Akyildiz and Perros [5] and by Onvural [31], whereas other blocking types, which are most common in communication systems, have been analyzed and compared by Van Dijk [38, 39]. Each blocking mechanism defines the service center blocking time, the behaviors of arriving customers to full capacity service center and the servers' activity in the network. Blocking types have been compared and equivalence properties derived for open queueing networks $[32,38,39]$, for tandem networks [6, 11] and for closed queueing networks [8, 38].

Exact product form solutions for the steady-state joint queue length probability distribution of queueing networks with blocking have been derived in the literature under special constraints, for different blocking mechanisms [1-4, 14, 15, 17, 19-21, 22, 26, 27 , $30,33,36,39,40-43]$. Several approximate solutions have been proposed for queueing networks with blocking. A survey of exact and approximate methods for closed queueing networks with blocking has been recently presented by Onvural in [31]. Open queueing networks with blocking and a bibliography on networks with finite capacity queues have been presented by Perros $[34,35]$.

In this paper, we present a survey of product form solutions of open and closed queueing networks with blocking and of equivalence properties among different blocking 
product form network models. The equivalence properties include relationships between open and closed product form queueing networks with different blocking mechanisms.

Equivalence properties between networks with different blocking types are defined in terms of exact state space transformation functions. By using such equivalence relationships between steady-state queue length distributions, it is possible both to extend product form solution and solution methods defined for a given blocking type model to the corresponding blocking type network model and to extend or to relate insensitivity properties for queueing networks with different blocking mechanisms.

A consequence of the results of equivalence is an extension of the class of product form networks with blocking which includes models in which different nodes work under different blocking mechanisms. These queueing networks can be used to represent complex systems, for example models of integrated computer-communication systems whose components have different blocking models (e.g., network links, controllers, and computer I/O subsystems with different types of blocking).

The paper is organized as follows. Section 2 introduces the queueing network model and the different blocking types. Section 3 surveys and classifies product-form networks with blocking and presents equivalence properties between product form networks with different blocking types. Finally, Section 4 presents the conclusions.

\section{The model}

We consider queueing networks with both single and multiple types of customers. Wefirsintroducmodelssumptionsndnotationforthegeneradase. Consider a queueing network with blocking formed by $\mathrm{M}$ service centers (or nodes) and with $\mathrm{C}$ classes of customers. The set of job classes can be partitioned into $\mathrm{R}$ disjoint sets $\mathrm{E}_{1}, \ldots, \mathrm{E}_{\mathrm{R}}$ called chains. Each chain can be either open or closed. The queueing network is open or closed if it is formed only by open or closed chains, respectively. The network is mixed if it consists of both open and closed chains.

If chain $r$ is closed then $N_{r}$ denotes the number of customers in the network in chain $r$, $1 \leq \mathrm{r} \leq \mathrm{R}$, and $\mathrm{N}=\square_{1 \leq \mathrm{r} \leq \mathrm{R}} \mathrm{N}_{\mathrm{r}}$ the total network population. If chain $\mathrm{r}$ is open, we assume Poisson exogenous arrival processes at node $\mathrm{i}, 1 \leq \mathrm{i} \leq \mathrm{M}, 1 \leq \mathrm{r} \leq \mathrm{R}$. Poisson arrival rate can be either load independent or load dependent, respectively denoted as $\square$ and $\square a(n), n \geq 0$, rates depending on the total number of customers in the entire network, or, for multichain networks, depending on the total network population $\mathrm{m}_{\mathrm{r}}$ in each chain $\mathrm{r}$, denoted by $\mathrm{a}_{\mathrm{r}}(\mathbf{m})$ 
and $\mathbf{m}=\left(m_{1}, \ldots, m_{R}\right), m_{1} \geq 0$, for each chain $r, 1 \leq r \leq R$. Functions a and $a_{r}$ are arbitrary and non negative, $1 \leq \mathrm{r} \leq \mathrm{R}$.

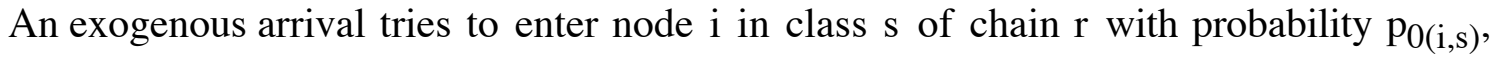
$1 \leq \mathrm{i} \leq \mathrm{M}, 1 \leq \mathrm{s} \leq \mathrm{C}, \mathrm{s} \square \mathrm{E}_{\mathrm{r}}, 1 \leq \mathrm{r} \leq \mathrm{R}$. In other words, the Poisson arrival process at node $\mathrm{i}$ in class $\mathrm{s}$ of chain $\mathrm{r}$ has parameter $\square \mathrm{p}_{0(\mathrm{i}, \mathrm{s})}$ for load independent arrivals, and $\square \mathrm{a}(\mathrm{n}) \mathrm{p}_{0(\mathrm{i}, \mathrm{s})}$ or $\square$ $\mathrm{a}_{\mathrm{r}}(\mathbf{m}) \mathrm{p}_{0(\mathrm{i}, \mathrm{s})}$, for load dependent arrivals, according to the arrival function definition.

Customers' behavior between service centers of the network is described by the routing matrix $P=\left\|p_{(i, s)(j, t)}\right\|, 1 \leq i, j \leq M, 1 \leq s, t \leq C$, where $p_{(i, s)(j, t)}$ denotes the probability that a job leaving class $s$ at node $i$ tries to enter node $j$ in class $t, t, s \square E_{r}, 1 \leq r \leq R$. If chain $r$ is open then $\mathrm{p}_{(\mathrm{i}, \mathrm{s}) 0}, 1 \leq \mathrm{i} \leq \mathrm{M}, 1 \leq \mathrm{s} \leq \mathrm{C}$, denotes the probability that a job leaving class $\mathrm{s}$ in node $\mathrm{i}$ leaves the network. By definition, the following relation holds, for $1 \leq \mathrm{i} \leq \mathrm{M}, 1 \leq \mathrm{s} \leq \mathrm{C}$ :

$$
\sum_{\mathrm{j}=1 \mathrm{t}=1}^{\mathrm{M}} \sum_{(\mathrm{i}, \mathrm{s})(\mathrm{j}, \mathrm{t})}+\mathrm{p}_{(\mathrm{i}, \mathrm{s}) 0}=1
$$

Let us introduce vector $\mathbf{x}$ with components $x_{i s}, 1 \leq i \leq M, 1 \leq s \leq C, s \square E_{r}, 1 \leq r \leq R$, which can be obtained by solving the following linear system for each chain :

$$
\mathrm{x}_{\mathrm{is}}=\square \mathrm{p}_{0(\mathrm{i}, \mathrm{s})}+\sum_{\mathrm{j}=1}^{\mathrm{M}} \sum_{\mathrm{t} \square \mathrm{E}_{\mathrm{r}}}^{\mathrm{M}} \mathrm{x}_{\mathrm{jt}} \mathrm{p}_{(\mathrm{j}, \mathrm{t})(\mathrm{i}, \mathrm{s})}
$$

Note that for each closed chain $\mathrm{r}$, by definition, $\mathrm{p}_{0(\mathrm{i}, \mathrm{s})}=\mathrm{p}_{(\mathrm{i}, \mathrm{s}) 0}=0$ for $\mathrm{s} \square \mathrm{E}_{\mathrm{r}}, 1 \leq \mathrm{r} \leq \mathrm{R}$, $1 \leq \mathrm{i} \leq \mathrm{M}$. Hence for each closed chain $\mathrm{r}$, linear system (1) is homogeneous and it does not provide a unique solution. For queueing networks without blocking component $\mathrm{x}_{\text {is }}$ represents the throughput of node $i$ and class $s$ of chain $r$, for open chains, whereas it represents the relative throughput or mean number of visits of customers at node $\mathrm{i}$ class $\mathrm{s}$ of chain $\mathrm{r}$ if the chain is closed, $\mathrm{s} \square \mathrm{E}_{\mathrm{r}}, 1 \leq \mathrm{r} \leq \mathrm{R}, 1 \leq \mathrm{i} \leq \mathrm{M}[23,28]$. For queueing network with blocking this meaning is not generally true. 
Network routing matrix $P$ is said to be reversible if $x_{i s} p_{(i, s)(j, t)}=x_{j t} p_{(j, t)(i, s)}$, and $\square \mathrm{p}_{0(\mathrm{i}, \mathrm{s})}=\mathrm{x}_{\mathrm{is}} \mathrm{p}_{(\mathrm{i}, \mathrm{s}) 0}, 1 \leq \mathrm{i}, \mathrm{j} \leq \mathrm{M}, 1 \leq \mathrm{s}, \mathrm{t} \leq \mathrm{C}[21]$.

Service center $i, 1 \leq i \leq M$, is described by the number of servers, the service time distribution and the service discipline. Let $\mathbf{S}_{\mathrm{i}}$ denote the state of node $\mathrm{i}$, from which one can derive vector $\mathbf{n}_{\mathrm{i}}=\left(\mathrm{n}_{(\mathrm{i}, 1)}, \mathrm{n}_{(\mathrm{i}, 2)}, \ldots, \mathrm{n}_{(\mathrm{i}, \mathrm{C})}\right)$ where component $\mathrm{n}_{(\mathrm{i}, \mathrm{s})}$ denotes the number of jobs in node $\mathrm{i}$ and in class $\mathrm{s}, 1 \leq \mathrm{s} \leq \mathrm{C}$. Node $\mathrm{i}$ state $\mathbf{S}_{\mathrm{i}}$ can include other components according to the node type (service discipline and service time distribution) and the blocking type. Let $n_{i}$ and $n_{i r}$ denote the total number of jobs in node $i$ and node $i$ chain $r$, $1 \leq \mathrm{i} \leq \mathrm{M}, 1 \leq \mathrm{r} \leq \mathrm{R}$, respectively.

The service time distribution of jobs at node $i$, class $s$ is denoted by $F_{i s}(t), t \geq 0$, $1 \leq \mathrm{i} \leq \mathrm{M}, 1 \leq \mathrm{s} \leq \mathrm{C}$, with mean value $1 / \mu_{\mathrm{is}}$, if it is load independent. Node $\mathrm{i}$ service rate for jobs of class $\mathrm{s}$ in chain $\mathrm{r}$ can be defined as dependent on the number of customers either in the whole node $\mathrm{i}, \mathrm{n}_{\mathrm{i}}$, or in node $\mathrm{i}$ and chain $\mathrm{r}, \mathrm{n}_{\mathrm{ir}}$, and is denoted by $\mu_{\mathrm{is}} \mathrm{f}_{\mathrm{i}}\left(\mathrm{n}_{\mathrm{i}}\right)$ and $\mu_{\mathrm{is}} \mathrm{f}_{\mathrm{ir}}\left(\mathrm{n}_{\mathrm{ir}}\right)$, respectively, $n_{i}, n_{i r} \geq 0$, where $f_{i}$ and $f_{i r}$ are arbitrary non negative functions, $1 \leq i \leq M, s \square E_{r}$, $1 \leq \mathrm{r} \leq \mathrm{R}$.

Service time distributions $F_{i s}(t), t \geq 0, \square s, \square i$, are assumed to be finite mixtures of Erlang distributions $[9,23,28]$. Let $r_{i s}(\square)$ denote the probability that a job in node $i$ and class $s$ has a residual service requirement of $\square$ exponential phases up to complete its service, $\square \geq 1$. The detailed definition of state $\mathbf{S}_{\mathrm{i}}$ includes pairs $\left(\mathrm{k}_{\mathrm{ip}}, \square_{\mathrm{ip}}\right), 1 \leq \mathrm{p} \leq \mathrm{n}_{\mathrm{i}}, 1 \leq \mathrm{i} \leq \mathrm{M}$, where $\mathrm{k}_{\mathrm{ip}}$ denotes the class of the job in position $\mathrm{p}$ of the queue, and $\square_{\mathrm{ip}}$ its residual service requirement .

Service disciplines are assumed to be defined as follows [12, 13, 21]. Let $\mathrm{p}$ denote a job position in node $i$ queue, when there are $n_{i}$ jobs, $1 \leq p \leq n_{i}, 1 \leq i \leq M$. Let $\square\left(p, n_{i}+1\right)$ denote the probability that an arriving job is placed in position $p$ when there are $n_{i}$ jobs in the queue, $1 \leq \mathrm{p} \leq \mathrm{n}_{\mathrm{i}}+1$, and let $\square_{\mathrm{i}}\left(\mathrm{p}, \mathrm{n}_{\mathrm{i}}\right)$ denote the fraction of service destined to the job in position $\mathrm{p}, 1 \leq \mathrm{p} \leq \mathrm{n}_{\mathrm{i}}, \mathrm{n}_{\mathrm{i}} \geq 0,1 \leq \mathrm{i} \leq \mathrm{M}$. By definition, the following relationship holds :

$$
\sum_{\mathrm{p}=1}^{\mathrm{n}_{\mathrm{i}}+1} \square\left(\mathrm{p}, \mathrm{n}_{\mathrm{i}}\right)=\sum_{\mathrm{p}=1}^{\mathrm{n}_{\mathrm{i}}} \square\left(\mathrm{p}, \mathrm{n}_{\mathrm{i}}\right)=1 \quad 1 \leq \mathrm{p} \leq \mathrm{n}_{\mathrm{i}}+1, \mathrm{n}_{\mathrm{i}} \geq 0,1 \leq \mathrm{i} \leq \mathrm{M}
$$

According to this definition a service discipline is said symmetric [21] or station balancing [12] if it satisfies the following condition

$$
\square\left(\mathrm{p}, \mathrm{n}_{\mathrm{i}}\right)=\square_{\mathrm{i}}\left(\mathrm{p}, \mathrm{n}_{\mathrm{i}}+1\right) \quad \square \mathrm{p}, \square \mathrm{n}_{\mathrm{i}}
$$

The set of service disciplines which can be defined by using this scheme includes several scheduling policies such as First Come First Service (FCFS), Last Come First Service preemptive resume (LCFS), Processor Sharing (PS) and Random service. FCFS and Random disciplines are not symmetric, while LCFS and PS are symmetric policies. 
Examples of service disciplines which cannot be represented by the above definition are any scheduling policies dependent on service time or on job priority.

In queueing networks with blocking additional constraints on the number of customers are included to represent different types of resource constraints in real systems, which correspond to definitions of different parameters.

In the first case, let $\mathrm{B}_{\mathrm{i}}$ denote the maximum queue length admitted at node $\mathrm{i}$ (i.e., the maximum buffer size), $1 \leq \mathrm{i} \leq \mathrm{M}$. Then the total number of jobs in node $\mathrm{i}, \mathrm{n}_{\mathrm{i}}$, is assumed to satisfy the constraint $n_{i} \leq B_{i}, 1 \leq i \leq M$. In multichain and multiclass networks $(C>1$ classes and $\mathrm{R}>1$ chains of customers) one can also define a chain $\mathrm{r}$ dependent maximum queue length at node $\mathrm{i}, \mathrm{B}_{\mathrm{ir}}, 1 \leq \mathrm{i} \leq \mathrm{M}, 1 \leq \mathrm{r} \leq \mathrm{R}$, or a class $\mathrm{s}$ dependent maximum queue length at node $\mathrm{i}, \mathrm{B}_{(\mathrm{i}, \mathrm{s})}, 1 \leq \mathrm{i} \leq \mathrm{M}, 1 \leq \mathrm{s} \leq \mathrm{C}$.

In the second case, let $\mathrm{B}_{\mathrm{W}}$ denote the maximum population admitted in a subnetwork W of the whole network. In certain cases, in order to represent particular system behaviors, a minimum population value $\mathrm{L}_{\mathrm{W}}$ for subnetwork $\mathrm{W}$ it is also introduced. In other words, the total population in subnetwork $\mathrm{W}, \mathrm{n}_{\mathrm{W}}=\square_{\mathrm{i} \square \mathrm{W}} \mathrm{n}_{\mathrm{i}}$, is assumed to satisfy constraints $\mathrm{L}_{\mathrm{W}} \leq \mathrm{n}_{\mathrm{W}} \leq \mathrm{B}_{\mathrm{W}}$.

\subsection{Blocking mechanisms}

Various blocking mechanisms or types that describe different behaviors of customer arrivals at a full capacity node and the servers' activity in the network have been defined in the literature. We now introduce the most commonly used five blocking mechanisms [5, 31, 38, 39].

The first three blocking types, Blocking After Service, Blocking Before Service and Repetitive Service Blocking, have been named and classified in [5, 31]. They arise because of the finite capacity of each service center of the network or maximum queue length for each chain $\mathrm{r}[5,30,31]$.

Let $b_{i s}\left(\mathbf{n}_{\mathrm{i}}\right)$ denote the blocking function, i.e., the probability that a class $\mathrm{s}$ job arriving at node $\mathrm{i}$, class $\mathrm{s}$ is accepted when $\mathbf{n}_{\mathrm{i}}$ is the state of the node, $1 \leq \mathrm{i} \leq \mathrm{M}, 1 \leq \mathrm{s} \leq \mathrm{C}$. The blocking function can, for example, be defined which may only depend on the total number of jobs in the node $\left(\mathrm{n}_{\mathrm{i}}\right)$, the number of class $\mathrm{s}$ jobs or in the chain.

An example of a simple blocking function for single class queueing networks which allows to define the maximum queue length $B_{i}$ for each node $i, 1 \leq i \leq M$, is defined as follows [19] : 


$$
\mathrm{b}_{\mathrm{i}}\left(\mathrm{n}_{\mathrm{i}}\right)=1 \quad \text { for } 0 \leq \mathrm{n}_{\mathrm{i}}<\mathrm{B}_{\mathrm{i}}, \quad \mathrm{b}_{\mathrm{i}}\left(\mathrm{B}_{\mathrm{i}}\right)=0 \quad 1 \leq \mathrm{i} \leq \mathrm{M}
$$

More generally, one can define

$$
0<b_{i}\left(n_{i}\right) \leq 1 \quad \text { for } 0 \leq n_{i}<B_{i}, \quad b_{i}\left(B_{i}\right)=0 \quad 1 \leq i \leq M
$$

as an arbitrary non negative load-dependent function which can be used to represent a flow control mechanism of node i input traffic.

A blocking function $b_{i s}\left(\mathbf{n}_{\mathrm{i}}\right)$ for multiclass multichain queueing networks has been defined [3] which depends on the total job number in node $i\left(n_{i}\right)$, node $i$ chain $r\left(n_{i r}\right)$, or node $i$ and class $\mathrm{s}\left(\mathrm{n}_{(\mathrm{i}, \mathrm{s})}\right)$, as follows:

$$
\mathrm{b}_{\text {is }}\left(\mathbf{n}_{\mathrm{i}}\right)=\mathrm{h}_{\mathrm{i}}\left(\mathrm{n}_{\mathrm{i}}\right) \mathrm{h}_{\mathrm{ir}}\left(\mathrm{n}_{\mathrm{ir}}\right) \mathrm{h}_{(\mathrm{i}, \mathrm{s})}\left(\mathrm{n}_{(\mathrm{i}, \mathrm{s})}\right) \quad 1 \leq \mathrm{i} \leq \mathrm{M}, 1 \leq \mathrm{s} \leq \mathrm{C}, 1 \leq \mathrm{r} \leq \mathrm{R},
$$

where functions $h_{i}, h_{i r}, h_{(i, s)}$ are arbitrary, except the following constraint : if $h_{i}(l)=0$ then $h_{i}(k)=0 \square k \geq 1$, and similarly for functions $h_{\text {ir }}$ and $h_{(i, s)}$.

Remark. Note that, by using blocking functions, the actual routing probabilities of the queueing network can be interpreted as state dependent probabilities, and they are obtained by combining the routing probability matrix $\mathrm{P}$ with blocking functions $\mathrm{b}_{\mathrm{is}}\left(\mathbf{n}_{\mathrm{i}}\right)$. Indeed, the actual probability that a job leaving node $i$ and class $s$ is accepted by node $\mathrm{j}$ in class $t$, when $\mathbf{n}_{\mathrm{j}}$ is the state of node $\mathrm{j}$, is given by $\mathrm{p}_{(\mathrm{i}, \mathrm{s})(\mathrm{j}, \mathrm{t})} \mathrm{b}_{\mathrm{jt}}\left(\mathbf{n}_{\mathrm{j}}\right), 1 \leq \mathrm{i}, \mathrm{j} \leq \mathrm{M}, 1 \leq \mathrm{s}, \mathrm{t} \leq \mathrm{C}, \square \mathbf{n}_{\mathrm{j}}$.

It is worth noting that also some queueing networks with state dependent routing, such as the model proposed in [37] and extended to multiclass networks in [25,41], can be included in this model definition, by appropriately defining the state dependent routing probabilities defined in terms of a constant factor and blocking functions.

The last two blocking mechanisms, Stop and Recirculate Blocking, which are very common in communication systems, have been named and compared in [38,39]. They arise because of the maximum queue length constraint for either a subnetwork or the total queueing network population .

Blocking After Service (BAS) (classical, transfer, manufacturing, production blocking, $[1,5,7,8,11,19,18,29,30-35])$ : if a job attempts to enter a full capacity queue $\mathrm{j}$ upon completion of a service at node $i$, it is forced to wait in node $i$ server, until the destination node $\mathrm{j}$ can be entered. The server of source node $\mathrm{i}$ stops processing jobs (it is blocked) until destination node $\mathrm{j}$ releases a job. In other words, node $\mathrm{i}$ service will be resumed as soon as a departure occurs from node $\mathrm{j}$. At that time the job waiting in node $\mathrm{i}$ immediately moves to node $\mathrm{j}$.

If more than one node is blocked by the same node $\mathrm{j}$, then a scheduling discipline must be considered to define the unblocking order of the blocked nodes when a departure occurs 
from node j. First Blocked First Unblocked is a possible discipline $[7,31]$ which states that the first node to be unblocked is the one which was first blocked.

This blocking mechanism has been used to model production systems and disk $\mathrm{I} / \mathrm{O}$ subsystems.

Blocking Before Service (BBS) (service, immediate blocking, [6, 8, 10, 11, 16, 17 3035]): when a destination node $\mathrm{j}$ becomes full, it blocks the service in each of its possible sending nodes $i$ (i.e., $\square$ i such that for some classes $s$ and $\left.t p_{(i, s)(j, t)}>0,1 \leq i \leq M, 1 \leq s, t, \leq C\right)$, provided that node $\mathrm{i}$ is servicing a job whose destination node is $\mathrm{j}$. Services will be resumed as soon as a departure occurs from node $\mathrm{j}$. The destination node of a blocked customer does not change, and it is declared before it starts receiving service.

Two different subcategories can be introduced [31] depending on whether the server can be used as service center buffer when the node is blocked:

BBS-SNO (server is not occupied) when the server of the blocked node cannot be used to hold a customer.

BBS-SO (server occupied) when the server of the blocked node is used to hold a customer.

A variant of the BBS type has been considered [8, 17, 24] when the overall set of sending nodes is blocked, and it is defined as follows :

BBS-O (Overall Blocking Before Service, type 4) : when a destination node $\mathrm{j}$ becomes full, it blocks the service in each of its possible sending nodes $i$, regardless of the destination of the currently processed job. Services will be resumed as soon as a departure occurs from node $\mathrm{j}$. The destination node of a blocked customer does not change.

This blocking mechanism has been used to model production, telecommunication, and computer systems.

Repetitive Service Blocking (RS) (rejection, retransmission, repeat protocol [2, 3, 4, 8, $11,14,15,19,20,22,30-35,36,38,41,42])$ : a job upon completion of its service at queue $\mathrm{i}$ attempts to enter destination queue $\mathrm{j}$. If node $\mathrm{j}$ is full, the job is looped back into the sending queue $\mathrm{i}$, where it receives a new independent service according to the service discipline.

Two different sub-categories have been introduced depending on whether the job, after receiving a new service, chooses a new destination node independently of the one that it had selected the previously:

RS-RD (random destination) if a job destination is randomly chosen at the end of each new service, independent of the previous choices. 
RS-FD (fixed destination) if a job destination is determined after the first service and can not be modified.

This blocking type has been used to model telecommunication systems.

For the following two blocking types the population either of a subnetwork or of the total network is assumed to be in the range [L,U], where $\mathrm{L}$ and $\mathrm{U}$ are the minimum and maximum populations admitted, respectively. This constraint can be represented by an appropriate definition of both the load dependent arrival rate functions $a(n), n \geq 0$ and of the (network) blocking function $\mathrm{d}(\mathrm{n}), \mathrm{n} \geq 0$. For multichain networks arrival and blocking functions can also be defined for each chain $r$, dependent on the total network population $\mathrm{m}_{\mathrm{r}}$ in chain $\mathrm{r}, \mathrm{a}_{\mathrm{r}}(\mathbf{m})$ and $\mathrm{d}_{\mathrm{r}}(\mathbf{m}), \mathbf{m}=\left(\mathrm{m}_{1}, \ldots, \mathrm{m}_{\mathrm{R}}\right) \geq 0,1 \leq \mathrm{r} \leq \mathrm{R}$.

STOP Blocking (interruption [38, 39]): the service rate of each node is delayed by a factor $d(n) \geq 1$, where $n \geq 0$ is the total network population. In other words, the actual job service rate of each node depends on the state of the entire network according to the function $d(n)$. When $d(n)=0$ then the service at each node in the network is stopped. Services will be resumed at each node as soon as an exogenous arrival occurs.

This blocking mechanism has been used to model communication systems.

RECIRCULATE Blocking (triggering protocol [26, 27, 38]): a job upon completion of its service at queue $i$ in class $s$ actually leaves the network with probability $p_{(i, s) 0} d(n)$, when $\mathrm{n}$ is the total network population, whereas it is forced to stay in the network with probability $\mathrm{p}_{(\mathrm{i}, \mathrm{s}) 0}[1-\mathrm{d}(\mathrm{n})]$, according to routing probabilities. Consequently, a job completing the service at node $\mathrm{i}$ class $\mathrm{s}$ actually enters node $\mathrm{j}$ in class $\mathrm{t}$ with state dependent routing probability

$$
\mathrm{p}_{(\mathrm{i}, \mathrm{s})(\mathrm{j}, \mathrm{t})}+\mathrm{p}_{(\mathrm{i}, \mathrm{s}) 0}[1-\mathrm{d}(\mathrm{n})] \mathrm{p}_{0(\mathrm{j}, \mathrm{t})} \quad 1 \leq \mathrm{i}, \mathrm{j} \leq \mathrm{M}, 1 \leq \mathrm{s}, \mathrm{t} \leq \mathrm{C}, \mathrm{n} \geq 0 .
$$

This blocking type has been used to model communication systems.

Queueing networks with blocking can deadlock, depending on the blocking type definition. If a deadlock occurs then either prevention techniques or detection and resolving techniques must be applied. Deadlock prevention for some types of blocking has been discussed [31].

Hereafter we shall consider deadlock-free queueing networks in steady-state conditions. 


\subsection{Product form solution}

The state of a queueing network can be represented by an $\mathrm{M}$-vector $\mathbf{S}=\left(\mathbf{S}_{1}, \ldots, \mathbf{S}_{\mathbf{M}}\right)$, where $\mathbf{S}_{\mathrm{i}}$ is the state of node $\mathrm{i}, 1 \leq \mathrm{i} \leq \mathrm{M}$. The state space $\mathrm{E}$ of the network is the set of all feasible states. Queueing network evolution can be represented by a continuous time ergodic Markov chain with discrete state space E. Under the hypothesis of irreducible routing matrix $\mathrm{P}$, there exists a unique steady-state queue length probability distribution $\boldsymbol{\pi}$ $=\{\pi(\mathbf{S}), \mathbf{S} \square \mathrm{E}\}$, which can be obtained by solving the following homogeneous linear system [23] :

$$
\boldsymbol{\pi} \mathrm{Q}=\mathbf{0}
$$

subject to the normalizing condition $\square \mathrm{S} \square \mathrm{E} \pi(\mathbf{S})=1$ and where $\mathrm{Q}$ is the process transition rate matrix.

Even if the joint queue length distribution $\pi$ of queuing networks with blocking can be obtained by formula (3), and other performance measures can be derived from $\boldsymbol{\pi}$, the solution of the linear system (3) becomes infeasible as the state space E dimension grows, proportionally to the parameters of the model including the number of customers, nodes and chains.

Under certain constraints, depending both on the network definition and the blocking mechanism used, $\pi$ has a product form solution $[1-4,14,15,17,19-21,22,26$, $27,30,33,36,39,40-43]$. We consider open, closed and mixed queueing networks analyzed in steady-state conditions.

For single class open or closed queueing networks with blocking, product form solutions can be defined as follows :

$$
\pi(\mathbf{S})=\frac{1}{\mathrm{G}} \mathrm{V}(\mathrm{n}) \square_{\mathrm{i}=1}^{\mathrm{M}} \mathrm{g}_{\mathrm{i}}\left(\mathrm{n}_{\mathrm{i}}\right)
$$

where $\mathrm{G}$ is a normalizing constant, $\mathrm{n}$ is the total network population, and the functions $\mathrm{V}$ and $g_{i}, 1 \leq i \leq M$, are defined in terms of network parameters, depending on the blocking type and additional constraints.

Similarly, for multichain open, closed or mixed queueing networks with blocking, formed by $\mathrm{M}$ nodes and $\mathrm{R}$ chains, product form solutions can be defined as follows:

$$
\pi(\mathbf{S})=\frac{1}{\mathrm{G}} \square_{\mathrm{r}=1}^{\mathrm{R}} \mathrm{V}_{\mathrm{r}}\left(\mathrm{m}_{\mathrm{r}}\right) \square_{\mathrm{i}=1}^{\mathrm{M}} \mathrm{g}_{\mathrm{i}}\left(\mathbf{n}_{\mathrm{i}}\right)
$$

where $\mathrm{G}$ is a normalizing constant, $\mathrm{m}_{\mathrm{r}}$ is the total network population in chain $\mathrm{r}, 1 \leq \mathrm{r} \leq \mathrm{R}$, and the functions $\mathrm{V}_{\mathrm{r}}, 1 \leq \mathrm{r} \leq \mathrm{R}$, and $\mathrm{g}_{\mathrm{i}}, 1 \leq \mathrm{i} \leq \mathrm{M}$, are defined in terms of network parameters, depending on the blocking type and additional constraints.

The complete definition of funtions $\mathrm{V}, \mathrm{V}_{\mathrm{r}}$ and $\mathrm{g}_{\mathrm{i}}$ in the different cases of product form networks with blocking will be given in detail in the next Section. 
Other performance indices of interest such as throughput, mean queue length and utilization, can be calculated from $\pi$. Let $\mathrm{L}_{\mathrm{i}}, \mathrm{U}_{\mathrm{i}}, \mathrm{U}_{\mathrm{i}}$ and $\mathrm{T}_{\mathrm{i}}$ denote the mean queue length, node utilization, throughput and mean response time of node $\mathrm{i}, 1 \leq \mathrm{i} \leq \mathrm{M}$, respectively.

\section{Equivalence properties in product-form blocking queueing networks}

In this Section, we survey product form solutions and equivalence properties of blocking networks reported in the literature.

For each network topology defined we present a theorem called "PF" (Product Form) to summarize the product form results and a theorem called "EP" (Equivalence Property) to summarize the equivalence properties between product form queueing networks with blocking. Note that in EP theorems, only the equivalencies which can be used to extend the class of product form solutions are reported.

The results presented in PF theorems hold for homogeneous networks, that is, all nodes work under the same blocking mechanism. Moreover the corollaries present product form solutions for non-homogeneous networks, which are deduced from PF and EP theorems.

In order to present the equivalence properties among different blocking models in terms of steady-state joint queue length distribution, we shall first introduce two basic relationships, defined both between two closed networks and between two open networks. First, we consider queueing networks with maximum queue length constraints either at node $\mathrm{i}, \mathrm{B}_{\mathrm{i}}$, or at node $\mathrm{i}$, chain $\mathrm{r}, \mathrm{B}_{\mathrm{ir}}, 1 \leq \mathrm{i} \leq \mathrm{M}, 1 \leq \mathrm{r} \leq \mathrm{R}$.

Consider two networks with identical parameters (M, C, R, $\square, a_{r}(\mathbf{m}), N_{r}, P, f_{i}\left(n_{i}\right)$, $\left.\square_{\mathrm{is}}, \square \mathbf{m}, \square \mathrm{n}_{\mathrm{i}}, \leq \mathrm{i} \leq \mathrm{M}, 1 \leq \mathrm{r} \leq \mathrm{R}, 1 \leq \mathrm{s} \leq \mathrm{C}\right)$, with buffer sizes $\mathrm{B}_{\mathrm{i}}$ and $\mathrm{B}_{\mathrm{i}}{ }^{\prime}$, or $\mathrm{B}_{\mathrm{ir}}$ and $\mathrm{B}_{\mathrm{ir}}{ }^{\prime}, 1 \leq \mathrm{i} \leq \mathrm{M}$, $1 \leq \mathrm{r} \leq \mathrm{R}$, respectively, and different blocking mechanisms $\mathrm{X}$ and $\mathrm{Y}$, respectively, where $\mathrm{X}, \mathrm{Y}$ $\square B M$, where $B M$ is the set of all blocking mechanisms defined in Section 2.

Note that, if the two networks are closed then $\square=\mathrm{a}_{\mathrm{r}}(\mathbf{m})=0,1 \leq \mathrm{r} \leq \mathrm{R}, \square \mathbf{m}$.

Let $\mathrm{E}$ and $\mathrm{E}^{\prime}$ be the state spaces of two networks and let $\square^{\mathrm{X}}=\left\{\square^{\mathrm{X}}(\mathbf{S}), \mathbf{S} \square \square\right\}$ and $\square^{\mathrm{Y}}=\left\{\square^{\mathrm{Y}}\left(\mathbf{S}^{\prime}\right), \mathbf{S}^{\prime} \square \mathrm{E}^{\prime}\right\}$ denote the corresponding steady-state probability distributions.

We introduce the following relations between the two blocking types $\mathrm{X}$ and $\mathrm{Y}$ :

- identity: blocking types $\mathrm{X}$ and $\mathrm{Y}$ are said to be identical, if $\mathrm{B}_{\mathrm{i}}=\mathrm{B}_{\mathrm{i}}{ }^{\prime}$ or $\mathrm{B}_{\mathrm{ir}}=\mathrm{B}_{\mathrm{ir}}{ }^{\prime}, 1 \leq \mathrm{i} \leq \mathrm{M}$, $1 \leq \mathrm{r} \leq \mathrm{R}$, and if $\mathrm{E}=\mathrm{E}^{\prime}$, and $\mathrm{\square}^{\mathrm{X}}=\square^{\mathrm{Y}}$.

- reducibility: blocking type $\mathrm{X}$ is said to be reducible to blocking type $\mathrm{Y}$ under one of the two following conditions: 
(i) if $B_{i}=B_{i}{ }^{\prime}$ or $B_{i r}=B_{i r}{ }^{\prime}, 1 \leq i \leq M, 1 \leq r \leq R$, and if there exists a function $f$ so that $\square^{\mathrm{X}}=\mathrm{f}\left(\square^{\mathrm{Y}}\right)$;

(ii) if there exist functions $g_{i}$ or $g_{i r}$ such that $B_{i}=g_{i}\left(B_{i}^{\prime}\right)$ or $\quad B_{i r}=g_{i r}\left(B_{i r}{ }^{\prime}\right), 1 \leq i \leq M$, $1 \leq \mathrm{r} \leq \mathrm{R}$, and if there exists a bijective function f between the network state spaces $\mathrm{E}$ and $\mathrm{E}^{\prime}$ so that steady-state probability is identical for corresponding states, i.e., $\square^{\mathrm{X}}(\mathbf{S})=\square^{\mathrm{Y}}(\mathrm{f}(\mathbf{S})), \square \mathbf{S} \square \square$ and $\mathrm{f}(\mathbf{S}) \square \mathrm{E}^{\prime}$.

We shall first present product form solution and equivalence properties for two node networks. Then we will consider some special topologies of closed networks, i.e., cyclic and central server topology networks. A more general class of networks is represented by the case of reversible routing. Finally, we discuss networks with arbitrary topologies.

\subsection{Two node cyclic networks}

In this Section we present product form results and equivalences for two node cyclic networks with blocking (see Fig. 2, with $M=2$ ). First we observe that, since each node has only one destination node, by definition, we have :

- RS-RD and RS-FD blocking are identical,

- BBS-SO and BBS-O blocking are identical.

For single class of jobs and FCFS exponential service centers, product form solution of the joint queue length equilibrium distribution has been proved under BBS-SO blocking in [17], under RS blocking in [19, 22, 36] and under BAS blocking in [1].

Cohen [14] proved that networks with multiple job classes and general service time distributions with processor sharing discipline and load dependent service rates have product form solutions under RS blocking. For networks with multiple job classes, general service time distribution at nodes with symmetric scheduling disciplines, product form solution has been proved by van Dijk and Tijms in [40] under RS blocking. The authors also proved an insensitivity property of the distribution of jobs which shows that the solution depends only on the mean of the service time distribution.

A similar product form result for multiclass networks with BCMP type nodes [9], and class independent buffer capacities $B_{i}, 1 \leq i \leq M$, has been proved by Onvural [30].

The following theorem summarizes product form results for homogeneous networks.

\section{Theorem PF1}


A homogeneous two-node closed cyclic queueing network with multiple classes of jobs, class independent buffer capacities $B_{i}, i=1,2$, and BCMP type nodes has the following product form solution of the joint queue length equilibrium distribution under $B A S, B B S-S O$, and RS blocking:

product form (5) with

$$
\begin{aligned}
& \mathrm{V}_{\mathrm{r}}\left(\mathrm{m}_{\mathrm{r}}\right)=1 \quad 1 \leq \mathrm{r} \leq \mathrm{R}, \square \mathrm{m}_{\mathrm{r}} \\
& \mathrm{g}_{\mathrm{i}}\left(\mathbf{n}_{\mathrm{i}}\right)=\mathrm{g}_{\mathrm{i}}\left(\mathrm{n}_{\mathrm{i}}\right) \prod_{\mathrm{r}=1}^{\mathrm{R}} \mathrm{g}_{\mathrm{ir}}\left(\mathrm{n}_{\mathrm{ir}}\right) \square_{\mathrm{s}=1}^{\mathrm{C}} \mathrm{g}_{(\mathrm{i}, \mathrm{s})}\left(\mathrm{n}_{(\mathrm{i}, \mathrm{s})}\right) \quad \square \mathbf{n}_{\mathrm{i}}, 1 \leq \mathrm{i} \leq \mathrm{M}
\end{aligned}
$$

where,

$$
\begin{aligned}
& g_{i}\left(n_{i}\right)=\prod_{1=1}^{n_{i}} \frac{r_{i k_{i 1}}\left(\square_{i 1}\right)}{f_{i}(l)} \\
& g_{i r}\left(n_{i r}\right)=1 \\
& g_{(i, s)}\left(n_{(i, s)}\right)=\left(x_{i s} / \mu_{i s}\right)^{n_{(i, s)}}
\end{aligned}
$$

The proof is based on the underlying Markov processes of the queueing networks which are reversible. In the theorem's proof, Onvural [30] shows that the product form solution of the equilibrium distribution under either BBS-SO or RS blocking is identical, up to a normalizing constant, to the product form equilibrium distribution of the same network with infinite buffer capacity queues, and it can be obtained by truncating the state space so that, at most, $B_{i}$ jobs are allowed at node $i, 1 \leq i \leq M$. Indeed, the Markov process of the blocking network is obtained by truncating the Markov process of the network with infinite buffer capacities. Hence, product form solution immediately follows from the theorem for truncated Markov processes of reversible Markov processes which states that the truncated process shows the same equilibrium distribution as the whole process normalized on the truncated sub-space [21].

A similar proof is given, in terms of joint queue length distribution, between a two node cyclic closed network under BAS blocking and the corresponding network with infinite buffer capacity queues [30]. In this case the state space of the underlying Markov process of the network with blocking is obtained by truncating the state space of the network without blocking, by assuming that at most $\mathrm{B}_{\mathrm{i}}+1$ jobs are admitted in node $\mathrm{i}$, $1 \leq \mathrm{i} \leq \mathrm{M}$. According to the notation introduced in Section 2 , let $\left(\mathbf{n}_{1}, \mathbf{n}_{2}\right)$ denote the state of the network. Let $\mathbf{n}_{\mathrm{i}} \mathrm{b}_{\mathrm{s}}$ denote node $\mathrm{i}$ state, when node $\mathrm{i}$ is blocked and the job in service is of class $\mathrm{s}, \mathrm{i}=1,2,1 \leq \mathrm{s} \leq \mathrm{C}$, and let $\mathbf{e}_{\mathrm{s}}$ denote the $\mathrm{C}$-vector with all zero components except 
for the s-th equal to 1 . Then the following theorem, which has been proved [30], states the equivalence properties which hold between blocking types RS, BBS-SNO and BBS-SO.

\section{Theorem EP1}

In a two node closed cyclic queueing network with multiple classes of jobs, class independent buffer capacities $B_{i}, i=1,2$, and BCMP type nodes the following equivalence properties hold:

i) BBS-SO and RS blocking mechanisms are identical,

ii) BBS-SO and BBS-SNO blocking mechanisms are identical if $N \leq B_{1}+B_{2}-2$,

iii) if service rates are load independent BAS blocking is reducible to BBS-SO blocking when $B_{i}{ }^{B B S-S O}=B_{i}{ }^{B A S}+1$ and the following function holds

$$
\begin{aligned}
& \pi^{\mathrm{BAS}}\left(\mathbf{n}_{1}, \mathbf{n}_{2}\right)=\pi^{\mathrm{BBS}-\mathrm{SO}}\left(\mathbf{n}_{1}, \mathbf{n}_{2}\right) \quad \square\left(\mathbf{n}_{1}, \mathbf{n}_{2}\right): \sum_{\mathrm{r}=1}^{\mathrm{R}} \mathrm{n}_{\mathrm{ir}} \leq \mathrm{B}_{\mathrm{ir}}^{\mathrm{BAS}} \quad \mathrm{i}=1,2 \\
& \pi^{\mathrm{BAS}}\left(\mathbf{n}_{1}, \mathbf{n}_{2} \mathrm{bs}_{\mathrm{s}}\right)=\pi^{\mathrm{BBS}-\mathrm{SO}}\left(\mathbf{n}_{1}+\mathbf{e}_{\mathrm{s}}, \mathbf{n}_{2}-\mathbf{e}_{\mathrm{s}}\right) \quad \square\left(\mathbf{n}_{1}, \mathbf{n}_{2}\right): \sum_{\mathrm{r}=1}^{\mathrm{R}} \mathrm{n}_{1 \mathrm{r}} \leq \mathrm{B}_{1 \mathrm{r}}^{\mathrm{BAS}} \\
& \pi^{\mathrm{BAS}}\left(\mathbf{n}_{1} \mathrm{~b}_{\mathrm{s}}, \mathbf{n}_{2}\right)=\pi^{\mathrm{BBS}-\mathrm{SO}_{(}}\left(\mathbf{n}_{1}-\mathbf{e}_{\mathrm{s}}, \mathbf{n}_{2}+\mathbf{e}_{\mathrm{s}}\right) \quad \square \quad\left(\mathbf{n}_{1}, \mathbf{n}_{2}\right) \quad \text { : }
\end{aligned}
$$

$\sum_{r=1}^{\mathrm{R}} \mathrm{n}_{2 \mathrm{r}} \leq \mathrm{B}_{2 \mathrm{r}}^{\mathrm{BAS}}$

By combining theorems FP1 and EP1 one can immediately derive the following result for non homogeneous networks.

\section{Corollary 1}

A non-homogeneous two node closed cyclic queueing network with multiple classes of jobs, class independent buffer capacities $B_{i}, i=1,2$, and BCMP type nodes shows the product form solution (pf1) of the joint queue length equilibrium distribution under BAS, BBS-SO and RS blocking.

The same networks with multiple job classes in which no class changes are allowed, and with class dependent buffer capacities $B_{i r}, 1 \leq i \leq M, 1 \leq r \leq R$, (see Fig. 1) have been considered by Onvural in [30], where a product form solution has been proved under particular constraints as follows.

\section{Theorem PF2}

A homogeneous two node closed cyclic queueing network, with multiple job classes and with class dependent buffer capacities $B_{i r}, i=1,2$ and $r=1,2, \ldots, R$, with 
BCMP nodes, except for processor sharing (PS) service discipline, shows a product form solution of the joint queue length equilibrium distribution under BBS-SO, BBS-SNO or RS blocking. When nodes with PS discipline are included, the blocking network shows the product form solution (pfl) if the service rate depends on the total number of jobs at that node, including both blocked and non-blocked jobs, as if there was no blocking.

Hence one can immediately derive the following equivalences between BBS-SO, BBS-SNO and RS blocking types, both for homogeneous and for non-homogeneous networks, as stated by the following theorem and corollary.

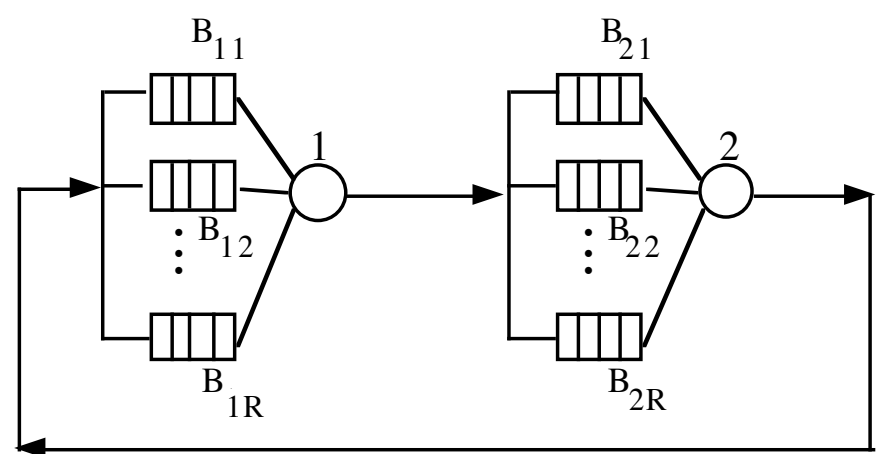

- Fig. 1 - A two-node closed queueing network with multiple job classes and class dependent buffer capacities -

\section{Theorem EP2}

In a two node closed cyclic queueing network with multiple job classes and with class dependent buffer capacities $B_{i r}, i=1,2$ and $r=1,2, \ldots, R$, and with BCMP nodes where the service rate at the nodes with PS discipline depends on the total number of jobs in the node, both blocked and non-blocked, BBS-SO, and RS blocking mechanisms are identical,.The resultt holds for $B B S-S N O$ if $N \leq B_{1}+B_{2}-2$.

\section{Corollary 2}

A non-homogeneous two node closed cyclic queueing network with multiple job classes and with class dependent buffer capacities $B_{i r}, i=1,2$ and $1 \leq r \leq R$, and with BCMP nodes where the service rate at the nodes with PS discipline depends on the total number of jobs in the node, both blocked and non-blocked, shows the product form solution (pfl) under BBS-SO and RS blocking, and if $N \leq B_{1}+B_{2}-2$ under BBS-SNO, too.. 
Equivalence properties for exponential two nodes networks with a single job class and load independent service rates have been proved in [8], by including equivalences in terms not only of joint queue length distribution but also of other mean performance indices, as given by the following theorem. For networks under BAS blocking, $\mathrm{n}_{\mathrm{i}}{ }^{\mathrm{b}}$ denotes that node $\mathrm{i}$ is blocked when there are $\mathrm{n}_{\mathrm{i}}$ jobs in it, $\mathrm{i}=1,2, \square \mathrm{n}_{\mathrm{i}}$.

\section{Theorem EP3}

For an exponential two node closed cyclic queueing network with single class of jobs and load independent service rates, the following equivalence properties hold:

i) BBS-SO and RS blocking mechanisms are identical. Moreover, these blocking mechanisms yield the same performance indices $\left(U_{i}, L_{i}, \square_{i}, T_{i}, i=1,2\right)$..

ii) BAS blocking is reducible to RS blocking, and the following function holds

$$
\begin{gathered}
\square^{\mathrm{BAS}}\left(\mathrm{n}_{1}, \mathrm{n}_{2}\right)=\text { Const } \square^{\mathrm{RS}}\left(\mathrm{n}_{1}, \mathrm{n}_{2}\right) \\
\square^{\mathrm{BAS}}\left(\mathrm{B}_{1},\left(\mathrm{~N}-\mathrm{B}_{1}\right)^{\mathrm{b}}\right)=\text { Const } \square^{\mathrm{RS}}\left(\mathrm{B}_{1}, \mathrm{~N}-\mathrm{B}_{1}\right) \square_{2} / \square_{1}
\end{gathered}
$$$$
\square^{\mathrm{BAS}}\left(\left(\mathrm{N}-\mathrm{B}_{2}\right)^{\mathrm{b}}, \mathrm{B}_{2}\right)=\text { Const } \square^{\mathrm{RS}}\left(\mathrm{N}-\mathrm{B}_{2}, \mathrm{~B}_{2}\right) \square_{1} / \square_{2}
$$

where Const is a normalizing constant.

Moreover, these blocking mechanisms yield the same performance in terms of node utilizations and throughputs, while having different mean queue lenghts and mean response times.

\subsection{Cyclic networks}

In this Section, we present product form results and equivalences for M-nodes closed cyclic networks with blocking (see Fig. 2). Similar to the two node networks, each node in M-node closed cyclic networks has only one destination node. Hence,

- RS-RD and RS-FD blocking are identical,

- BBS-SO and BBS-O blocking are identical.

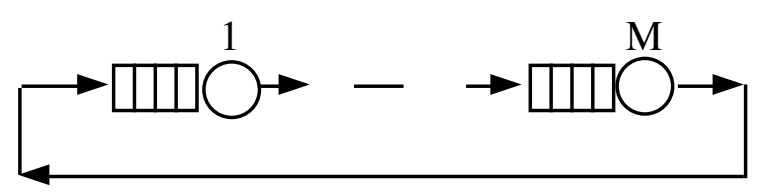

- Fig. 2 - M-node closed cyclic network -

Similarly, tandem networks under RS blocking were analyzed by Caseau and Pujolle in [11] with FCFS exponential service centers, intermediate arrivals and load dependent service rates. The authors showed that RS and BBS-SO blocking types are 
identical, and BAS and BBS-SNO are reducible when $\mathrm{B}_{\mathrm{i}}{ }^{\mathrm{BBS}-\mathrm{SNO}}=\mathrm{B}_{\mathrm{i}}{ }^{\mathrm{BAS}}+1,1 \leq \mathrm{i} \leq \mathrm{M}$. A similar result has been derived for closed cyclic networks, as reported by the following theorem EP4.

Closed cyclic networks with a single job class and FCFS exponential service centers with load independent service rates was first studied under BBS-SO blocking by Gordon and Newell in [17], and then under RS blocking by Hordijk and Van Dijk in [19]. Product form solution of the joint queue length distribution has been derived under the condition that no node can be empty. Product form results are sumarized in the following theorem PF3.

Equivalence properties among product form networks with blocking types BBS-SO, BBS-SNO, RS and BAS have been proved in [8] and in [31], and are sumarized in theorem EP4.

\section{Theorem PF3}

A homogeneous closed cyclic queueing network with a single job class, exponential service centers, load independent service rates, and with

$$
\mathrm{N} \geq \sum_{\mathrm{i}=1}^{\mathrm{M}} \mathrm{B}_{\mathrm{i}}-\min \left\{\mathrm{B}_{\mathrm{j}}, \mathrm{j}=1, \ldots, \mathrm{M}\right\}
$$

shows the following product form solution under BBS-SO or RS blocking mechanisms:

product form (4) with

$$
\begin{array}{ll}
\mathrm{V}(\mathrm{n})=1 & \square \mathrm{n} \\
\mathrm{g}_{\mathrm{i}}\left(\mathrm{n}_{\mathrm{i}}\right)=1 / \mathrm{C}^{\mathrm{n}_{\mathrm{i}}} & \square \mathrm{n}_{\mathrm{i}}, 1 \leq \mathrm{i} \leq \mathrm{M}
\end{array}
$$

where $\square=\left(\square_{l}, \ldots, \square_{M}\right)$ is obtained by solving system $\square=\square P^{\prime}$ where $P^{\prime}=\left\|p_{i j}^{\prime}\right\|$, $1 \leq i, j \leq M, p_{i j}^{\prime}=\square_{j} p_{j i}$ for $i \neq j$, and $p_{i i}^{\prime}=1-\sum_{j \neq i} p_{i j}^{\prime}$.

\section{Theorem EP4}

In a closed cyclic queueing network with a single job class, exponential service centers, load independent service rates, the following equivalence properties hold:

i) BBS-SO and RS blocking mechanisms are identical . Moreover, these blocking mechanisms yield the same mean performance indices $\left(U_{i}, L_{i}, \square_{i}, T_{i}, 1 \leq i \leq M\right)$.

ii) BBS-SO and BBS-SNO blocking mechanisms are identical if $N \leq \min \left\{B_{i}+B_{j}\right.$ with $\left.p_{i j}>0, i, j=1, \ldots, M\right\}-1$

Then, these blocking mechanisms yield the same performance indices $\left(U_{i}, L_{i}, \square_{i}\right.$, $\left.T_{i}, 1 \leq i \leq M\right)$. 
iii) $B B S-S N O$ and $B A S$ blocking are reducible when $B_{i}^{B B S-S N O}=B_{i}{ }^{B A S}+1$, $1 \leq i \leq M$, and the bijective function between the equilibrium distributions $\square^{B A S}$ and $\square^{B B S-S N O}$ is defined as follows :

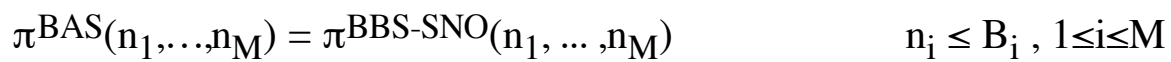

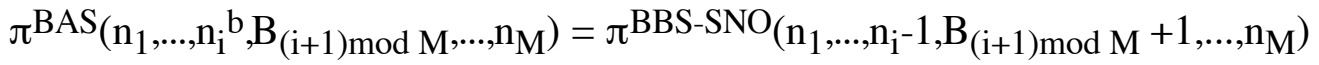

$$
\begin{aligned}
& 1 \leq \mathrm{i} \leq \mathrm{M}
\end{aligned}
$$

By combining the product form and the equivalence theorems PF3 and EP4 one can derive the following corollary which summarizes the product form results for nonhomogeneous cyclic networks.

\section{Corollary 4}

A non-homogeneous closed cyclic queueing network with a single job class, exponential service centers, load independent service rates, and with $N \geq \sum_{i=1}^{M} B_{i}-\min \left\{B_{j}, j=1, \ldots, M\right\}$, shows the product form solution (pf2) under BBS-SO and RS blocking mechanisms.

\subsection{Central server networks}

The central server model, shown in Fig. 3, is a reversible routing queueing network and we refer to the following Section for the product form solutions proved for the class of reversible routing networks. In this Section we survey results specifically proved only for central server networks.

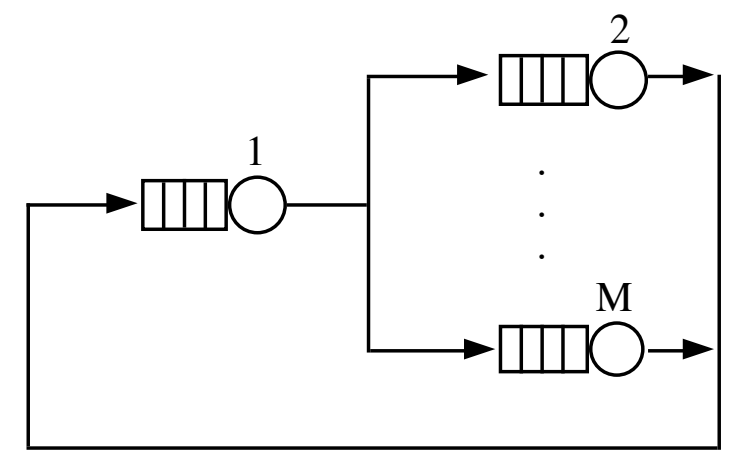

- Fig. 3 - A central server queueing network - 
First note that, for this network topology, each node except the central node has only one destination node, as for M-node cyclic networks. By definition, the following identities hold when only the central node has finite capacity queue:

- RS-RD and RS-FD blocking are identical,

- BBS-SO and BBS-O blocking are identical.

Central server networks have been studied under RS-RD blocking. A first product form equilibrium distribution was proved by Dallery and Yao in [15] for a single job class and general service time distribution at nodes with symmetric scheduling discipline. On the other hand, Towsley [37] proved a product form solution for a class of networks which includes the central server model with single job class, state-dependent routing and general service time distribution at nodes with symmetric scheduling discipline. However, as pointed out in Section 2, and as discussed and extended by Krzesinski [25] and by Yao and Buzacott to multiple job classes [41], there exists a relationship between state dependent routing and blocking functions. Therefore, such product form solution for networks with state dependent routing can be interpreted as blocking networks. The following theorem presents a generalization of these results obtained by combining state dependent routing and finite capacity queues derived by Akyildiz and von Brand [2]. Let 1 denote the central node.

\section{Theorem PF4}

Closed central server networks with

- multiplejob classes, but with the class type of a job fixed in the system, i.e., with $C=R$;

- state-dependent routing depending on the class type s defined as follows

$$
\begin{array}{ll}
p_{(1, s)(j, s)}(\boldsymbol{S})=w_{j s}\left(n_{(j, s)}\right) w_{s}\left(N_{s}-n_{(1, s)}\right) & \square \boldsymbol{S} \\
p_{(1, s)(j, s)}=1 & 2 \leq j \leq M, 1 \leq s \leq C
\end{array}
$$

- blocking functions for node $i$ and class $s$

$b_{i s}\left(\boldsymbol{n}_{i}\right)=h_{i}\left(n_{i}\right) h_{i s}\left(n_{(i, s)}\right) \quad \square \boldsymbol{n}_{i}, l \leq j \leq M, 1 \leq s \leq C$

- general service time distribution at nodes with symmetric scheduling

discipline and exponential service times distributions, equal for each class at the same node, when the scheduling is arbitrary

shows the following product form equilibrium distribution under RS-RD blocking:

product form (5) with

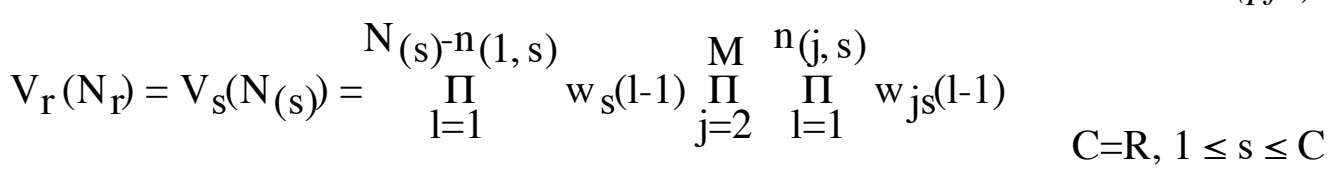




$$
\mathrm{g}_{\mathrm{i}}\left(\mathbf{n}_{\mathrm{i}}\right)=\mathrm{g}_{\mathrm{i}}\left(\mathrm{n}_{\mathrm{i}}\right) \underset{\mathrm{s}=1}{\mathrm{C}} \mathrm{g}_{(\mathrm{i}, \mathrm{s})}\left(\mathrm{n}_{(\mathrm{i}, \mathrm{s})}\right) \quad \square \mathbf{n}_{\mathrm{i}}, 1 \leq \mathrm{i} \leq \mathrm{M}
$$

with

$$
\begin{gathered}
\mathrm{g}_{\mathrm{i}}\left(\mathrm{n}_{\mathrm{i}}\right)=\square_{\mathrm{l}=1}^{\mathrm{n}_{\mathrm{i}}} \frac{\mathrm{h}_{\mathrm{i}}(\mathrm{l}-1)}{\mathrm{f}_{\mathrm{i}}(\mathrm{l})} \mathrm{r}_{\mathrm{i} \mathrm{k}}\left(\square_{\mathrm{i} 1}\right) \\
\mathrm{g}_{(\mathrm{i}, \mathrm{s})}\left(\mathrm{n}_{(\mathrm{i}, \mathrm{s})}\right)=\prod_{\mathrm{l}=1}^{\mathrm{n}_{(\mathrm{i}, \mathrm{s})}} \frac{\mathrm{h}_{(\mathrm{i}, \mathrm{s})}(1-1)}{\square_{\mathrm{is}}}
\end{gathered}
$$

with $n_{(i, s)}$ jobs of class $s$ in node $i, 1 \leq i \leq M$, and $N_{(s)}=\square_{1 \leq s \leq C} n_{(i, s)}$ the total number of jobs of class $s$ in the network, $1 \leq s \leq C$.

The following equivalence property is an application to the central server network of the more general theorem EP7 presented in Section 3.5. Moreover, note that these results can easily be extended to the multiclass case.

\section{Theorem EP5}

RS-RD and BBS-SO blocking mechanisms are identical when applied to central server networks with a single job class and where only the central node has finite capacity queue.

Moreover, these blocking mechanisms yield the same mean performance indices $\left(U_{i}, L_{i}\right.$, $\left.\square_{i}, T_{i}, 1 \leq i \leq M\right)$.

By combining theorems PF4 and EP5, a case of product form solution holds for central server networks with BBS-SO, as proved in [8].

\section{Corollary 5}

- An exponential central server network with a single job class and where only the central node has a finite buffer, shows product form solution (pf3) under BBS-SO blocking.

- A non-homogeneous exponential central server network with a single job class, where each node has BBS-SO blocking except the central one which has RS blocking, shows product form solution (pf3). 


\subsection{Reversible routing networks}

Queueing networks with reversible routing have been studied under RS-RD and STOP blocking. First, a product form solution has been proved for closed reversible networks with single class of jobs, FCFS exponential service centers and RS-RD blocking in $[19,22]$. Pittel [36] extended the product form solution to exponential closed queueing networks formed by nodes with PS discipline, with reversible routing and with multiple job classes but without class change and RS-RD blocking. Product form solution has been extended to multiclass mixed queueing networks with class independent buffer capacities and arbitrary service time distributions when the scheduling of the queue is symmetric [30, 42, 43], for RS-RD blocking. For similar networks, but with single job class, product form solution has been derived [38] for RS-RD and STOP blockings. Recently, this class of product form queueing networks with RS-RD blocking has been extended [3] to include more general blocking functions which can depend both on the total population, class population and routing chain population at the node. The following theorem summarizes such product form results for reversible routing networks.

\section{Theorem PF5}

A homogeneous open, closed or mixed network with

- reversible routing,

- multiple job classes,

- arbitrary service time distributions when the scheduling is symmetric and

exponential service time distributions, equal for each class at the same node, when the scheduling is not symmetric

- blocking functions for node $i$ and class $s$

$b_{i s}\left(\boldsymbol{n}_{i}\right)=h_{i}\left(n_{i}\right) h_{i r}\left(n_{i r}\right) h_{(i, s)}\left(n_{i, s)}\right) \quad \square \boldsymbol{n}_{i}$

when there are a total of $n_{i}$ jobs in node $i$, of which $n_{(i, s)}$ are in class $s$ and

$n_{\text {ir }}$ are in chain $r, 1 \leq i \leq M, 1 \leq r \leq R, 1 \leq s \leq C$,

shows the following product form solution under RS-RD blocking:

product form (5) with

$(p f 4)$

$$
\begin{aligned}
& \mathrm{V}_{\mathrm{r}}\left(\mathrm{m}_{\mathrm{r}}\right)=1 \quad \square \mathrm{m}_{\mathrm{r}} \geq 0,1 \leq \mathrm{r} \leq \mathrm{R} \\
& \mathrm{g}_{\mathrm{i}}\left(\mathbf{n}_{\mathrm{i}}\right)=\mathrm{g}_{\mathrm{i}}\left(\mathrm{n}_{\mathrm{i}}\right) \square_{\mathrm{r}=1}^{\mathrm{R}} \mathrm{g}_{\mathrm{ir}}\left(\mathrm{n}_{\mathrm{ir}}\right) \prod_{\mathrm{s}=1}^{\mathrm{C}} \mathrm{g}_{(\mathrm{i}, \mathrm{s})}\left(\mathrm{n}_{(\mathrm{i}, \mathrm{s})}\right)
\end{aligned}
$$

where 


$$
\begin{aligned}
& \mathrm{g}_{\mathrm{i}}\left(\mathrm{n}_{\mathrm{i}}\right)=\prod_{\mathrm{l}=1}^{\mathrm{n}_{\mathrm{i}}} \frac{\mathrm{h}_{\mathrm{i}}(\mathrm{l}-1)}{\mathrm{f}_{\mathrm{i}}(\mathrm{l})} \mathrm{r}_{\mathrm{i}} \mathrm{k}_{\mathrm{il}}\left(\square_{\mathrm{i} l}\right) \\
& \mathrm{g}_{\mathrm{ir}}\left(\mathrm{n}_{\mathrm{ir}}\right)=\prod_{\mathrm{l}=1}^{\mathrm{n}_{\mathrm{ir}}} \mathrm{h}_{\mathrm{ir}}(\mathrm{l}-1) \\
& \mathrm{g}_{(\mathrm{i}, \mathrm{s})}\left(\mathrm{n}_{(\mathrm{i}, \mathrm{s})}\right)=\prod_{\mathrm{l}=1}^{\mathrm{n}_{(\mathrm{i}, \mathrm{s})}} \frac{\mathrm{x}_{\mathrm{is}} \mathrm{h}_{(\mathrm{i}, \mathrm{s})}(1-1)}{\square_{\mathrm{is}}}
\end{aligned}
$$

The same network but with single job class, shows the following product form solution under STOP blocking:

$$
\begin{aligned}
& \text { productform (4) with } \\
& \mathrm{V}(\mathrm{n})=1 \quad \square \mathrm{n} \\
& \mathrm{g}_{\mathrm{i}}\left(\mathrm{n}_{\mathrm{i}}\right)=\left(\mathrm{x}_{\mathrm{i}} / \mu_{\mathrm{i}}\right)^{\mathrm{n}_{\mathrm{i}}} \quad \square \mathrm{n}_{\mathrm{i}} \geq 0,1 \leq \mathrm{i} \leq \mathrm{M}
\end{aligned}
$$

Note that in [38] a different state notation is considered, with one component for each job in the system, which denotes the node at which the job is present.

The following equivalence result between RS-RD and STOP blocking mechanisms has been proved in [38].

\section{Theorem EP6}

In a closed or open network with

- reversible routing,

- single job class,

- arbitrary service time distributions when the scheduling discipline is

symmetric and exponential service time distributions, equal for each class at the same node, when the scheduling is not symmetric,

$R S-R D$ and STOP blocking mechanisms are identical.

From theorems PF5 and EP6 one can immediately derive the following product form solution for non-homogeneous networks.

\section{Corollary 6}

A non-homogeneous mixed network with

- reversible routing,

- single job class, 
- arbitrary service tims distributions when the scheduling discipline is

symmetric and exponential service times distributions, equal for each

class at the same node, when the scheduling is arbitrary,

shows product form (pf5) solution under RS-RD and STOP blocking.

A more general network model has been considered in [4]. The authors consider networks of parallel queues with multiple job classes and reversible routing, where general interdependent blocking functions and general interdependent service functions are allowed. Formally, each service center i contains a set of queues and a job entering node $\mathrm{i}$ requires service at queue $q(i, s)$ depending on its present class $s, 1 \leq i \leq M, 1 \leq s \leq C$. This request is accepted with probability $\mathrm{A}_{\mathrm{i}}\left(\mathrm{q}(\mathrm{i}, \mathrm{s}) \mid \mathbf{n}_{\mathrm{i}}\right)$, when $\mathbf{n}_{\mathrm{i}}$ is the state of node $\mathrm{i}$, that is, the blocking probability depends not only on the total number of class s jobs, but also on the number of jobs of the other classes. The rate at which queue $q$ provides service is given by $f_{i}\left(q(i, s) \mid \mathbf{n}_{i}\right)$, that is, the rate out of one queue depends on the number of jobs in the entire node, including the other queues; the mean service rate of a job of class $s$ at queue $\mathrm{q}$ is denoted by $\square_{\mathrm{iq}}$. The network under RS-RD blocking shows a product form solution if both the reversible routing and an invariance condition hold. Informally, such an invariance condition requires that function $\mathrm{P}_{\mathrm{i}}\left(\mathbf{n}_{\mathrm{i}}\right)$, defined as follows

$$
\mathrm{P}_{\mathrm{i}}\left(\mathbf{n}_{\mathrm{i}}\right)=\prod_{\mathrm{k}=1}^{\mathrm{n}_{\mathrm{i}}} \frac{\mathrm{A}_{\mathrm{i}}\left(\mathrm{q}\left(\mathrm{i}, \mathrm{s}_{\mathrm{k}}\right)\left|\mathbf{n}_{\mathrm{i}}(\mathrm{k}-1)\right|\right.}{\mathrm{f}_{\mathrm{i}}\left(\mathrm{q}\left(\mathrm{i}, \mathrm{s}_{\mathrm{k}}\right)\left|\mathbf{n}_{\mathrm{i}}(\mathrm{k})\right|\right.} \quad \square \mathbf{n}_{\mathrm{i}}, 1 \leq \mathrm{i} \leq \mathrm{M}
$$

where $\mathbf{n}_{\mathrm{i}}(\mathrm{k})$ is state $\mathbf{n}_{\mathrm{i}}$ with $\mathrm{k}$ jobs in node $\mathrm{i}, 1 \leq \mathrm{i} \leq \mathrm{M}$, is invariant to the arriving order of jobs at node i, with respect to their class. See [4] for further details.

\section{Theorem PF6}

A homogeneous closed network with stations having parallel queues and

- reversible routing,

- multiple job classes,

- arbitraryerviceimesdistributionwhentheschedulings symmetric and exponentialservicetimesdistributionsequalfor eachclassatthesame node, when the scheduling is arbitrary

- interdependent blocking probability and service rate

shows the following product form solution under RS-RD blocking if the interdependent blocking and servicing satisfy the invariant condition : 
product form (5) with

$$
\begin{aligned}
& \mathrm{V}_{\mathrm{r}}\left(\mathrm{m}_{\mathrm{r}}\right)=1 \quad \square \mathrm{m}_{\mathrm{r}} \geq 0,1 \leq \mathrm{r} \leq \mathrm{R} \\
& \mathrm{g}_{\mathrm{i}}\left(\mathbf{n}_{\mathrm{i}}\right)=\mathrm{P}_{\mathrm{i}}\left(\mathbf{n}_{\mathrm{i}}\right) \square_{\mathrm{s}=1}^{\mathrm{C}} \mathrm{x}_{\mathrm{is}}^{\mathrm{n}_{(\mathrm{i}, \mathrm{s})}} \square_{\mathrm{q} \square \mathrm{s}}\left(\frac{1}{\square_{\mathrm{iq}}}\right)^{\mathrm{m}_{\mathrm{iq}}} \square_{\mathrm{s}: \mathrm{q}(\mathrm{i}, \mathrm{s}) \square \mathrm{s}}\left(\frac{1}{\square_{\mathrm{is}}}\right)^{\mathrm{n}(\mathrm{i}, \mathrm{s})}
\end{aligned}
$$

where $S$ is the set of symmetric queues, $m_{i q}$ is the number of jobs at node i in queue $q$, $\square q, 1 \leq i \leq M$.

\subsection{Arbitrary topology networks}

In this Section, we survey product form blocking networks without restrictions as to the topology, i.e., with arbitrary topologies.

The first result was obtained by Hordijk and Van Dijk in [19] for closed networks with a single job class, FCFS exponential service centers with load independent service rates and with RS-RD blocking, by assuming that the number of customers in the network is such that a node cannot be empty.

\section{Theorem PF7}

A homogeneous closed queueing network with a single job class, FCFS exponential service centers, load independent service rates and with

$$
N>\sum_{i=1}^{M} B_{i}-\min \left\{B_{j}, j=1, \ldots, M\right\}
$$

has the product form solution (pf2) under RS-RD blocking.

An equivalence property between RS-RD and BBS-SO blocking models for arbitrary topology exponential networks with single class of jobs has been proved in [8]. The extension to multiclass networks can easily be proved.

\section{Theorem EP7}

$R S-R D$ and BBS-SO blocking mechanisms are identical when applied to closed exponential queueing networks with single job class, where each node $i$ with finite buffer $\left(B_{i}<N\right)$ satisfies the following condition:

$$
\text { if } p_{j i}>0 \text { then } p_{j i}=1, \quad 1 \leq j \leq M
$$

In other words, condition (7) requires that each node with finite capacity is the only destination node for each upstream node. 
From such an equivalence property and from theorem PF7 the following product form solution for non-homogeneous networks with arbitrary topology can easily be proved.

\section{Corollary 7}

A non-homogeneous closed queueing network with a single job class, FCFS exponential service centers, load independent service rates and where each node with finite buffer satisfies condition (6), has the product form solution (pf2) under RS-RD and BBS-SO blocking mechanisms, if nodes with BBS-SO blocking satisfy condition (7).

Multiclass networks with FCFS exponential nodes under BAS blocking have been considered by Onvural and Perros in [33]. For these networks, a product form solution has been proved when the population is kept under an upper bound.

\section{Theorem PF8}

A homogeneous closed network with multiple job classes, class independent buffer capacities $B_{i}, 1 \leq i \leq M$, and FCFS exponential centers, shows the following product form solution of the joint queue length equilibrium distribution under BAS blocking, if $\sum_{r \sqcap R_{i}} N_{r}=\min \left\{B_{i}, 1 \leq i \leq M\right\}+1$

where $R_{i}$ is the set of routing chains that visit node $i, 1 \leq i \leq M$ :

product form (5) with

$$
\begin{aligned}
& \mathrm{V}_{\mathrm{r}}\left(\mathrm{m}_{\mathrm{r}}\right)=1 \quad \square \mathrm{m}_{\mathrm{r}}, 1 \leq \mathrm{r} \leq \mathrm{R} \\
& \mathrm{g}_{\mathrm{i}}\left(\mathbf{n}_{\mathrm{i}}\right)=\square_{\mathrm{s}=1}^{\mathrm{C}}\left(\begin{array}{l}
\mathrm{x}_{\mathrm{is}} \\
\square_{\mathrm{is}}
\end{array}\right)^{\mathrm{n}_{(\mathrm{i}, \mathrm{s})}}
\end{aligned}
$$

To our knowledge, no equivalence property has been proved between arbitrary topology networks with BAS blocking and other blocking mechanisms, and consequently no extensions of the product form solution to non-homogeneous networks can be derived.

RECIRCULATE blocking mechanism has been considered for arbitrary topology open networks with FCFS exponential nodes and Poisson arrivals [26, 27]. Jackson derived a product form solution for this blocking mechanism under a minimal population constraint. Lam extended this product form solution to multiclass networks with class interdependent blocking. 
STOP and RECIRCULATE blocking models have been analyzed and compared [39]. Van Dijk considers Jackson queueing networks with multiple job class having the job class-type fixed throughout its residence in the system, i.e., with $\mathrm{C}=\mathrm{R}$. The network population is kept within a range defined by a lower and an upper bound.

Both a product form solution and an equivalence property between STOP and RECIRCULATE blocking have been derived [39] as stated by the following theorems. Note that the product form solution proved for IS nodes can be easily extended to other service disciplines such as PS, LCFS and FCFS, as discussed in [39].

\section{Theorem PF9}

A homogeneous Jackson open network with multiple job classes and with the job class-type fixed throughout its residence in the system $(R=C)$, has the following product form solution under both STOP and RECIRCULATE blocking mechanisms:

product form (5) with

$$
\begin{aligned}
& \square=1 \\
& \mathrm{~V}_{\mathrm{r}}\left(\mathrm{m}_{\mathrm{r}}\right)=\prod_{\mathrm{l}=0}^{\mathrm{m}_{\mathrm{r}}-1} \mathrm{a}_{\mathrm{r}}(\mathrm{l}) \quad \square \mathrm{m}_{\mathrm{r}} \geq 0,1 \leq \mathrm{r} \leq \mathrm{R} \\
& \left.\mathrm{g}_{\mathrm{i}}\left(\mathbf{n}_{\mathrm{i}}\right)=\prod_{\mathrm{r}=1}^{\mathrm{R}} \frac{1}{\mathrm{n}_{\mathrm{ir}} !}\left(\mathrm{x}_{\mathrm{ir}}\right)^{\mathrm{D}_{\mathrm{ir}}}\right)^{\mathrm{ir}} \quad \square \mathbf{n}_{\mathrm{i}} \geq 0,1 \leq \mathrm{i} \leq \mathrm{M}
\end{aligned}
$$

\section{Theorem EP8}

In a Jackson open network with multiple job classes but with the class-type of a job fixed throughout its residence in the system, STOP and RECIRCULATE blocking mechanisms are identical.

Note that from the definitions of STOP and RECIRCULATE blocking mechanisms, defining a non-homogeneous network and then extending product form results it is not immediate.

Let us now consider an open Jackson network with a population constraint for lower and upper bounds. It is known that any open queueing network with finite queues and Poisson arrivals, can be exactly analyzed as a closed queueing network [31]. This closed queueing network will be called the "anologue" closed network of the open 
network . In the following we define an open Jackson network and its analogue closed one.

Let us consider an open Jackson network with parameters $\left(\mathrm{M}, \mathrm{\square}, \mathrm{a}(\mathrm{n}), \mathrm{P}, \mathrm{B}_{\mathrm{i}}, \square_{\mathrm{i}}\right.$, $1 \leq \mathrm{i} \leq \mathrm{M})$ with $\mathrm{F} 1$ and the following contraint on the network population $\mathrm{n}: \mathrm{L} \leq \mathrm{n} \leq \mathrm{U}, \mathrm{L}$, $U \geq 0$, and with $B_{i} \geq U, 1 \leq i \leq M$. Let $P=\left\|p_{i j}\right\| 0 \leq i, j \leq M$ be the $(M+1) x(M+1)$ routing matrix where $p_{i j}$ is the probability that upon service completion at node $i$ a job routes to station $j$, $1 \leq \mathrm{i}, \mathrm{j} \leq \mathrm{M}$, while $\mathrm{p}_{\mathrm{i} 0}$ is the probability that upon service completion at node $\mathrm{i}$ a job leaves the system and $\mathrm{p}_{0 \mathrm{i}}$ is the probability that an arriving job is routed to node $\mathrm{i}$, with $1 \leq \mathrm{i} \leq \mathrm{M}$, and $\mathrm{p}_{00}=0$.

The arrival rate function $a(n)$ and the departure blocking function $d(n)$, when $n$ jobs are present in the entire network, satisfy the following contraints [39]:

$$
\begin{aligned}
& a(U)=0 \text { if } U<\infty \text { and } a(n)>0, \text { for } L \leq n<U, \\
& d(L)=0 \text { if } L>0 \text { and } d(n)=1, \text { for } L<n \leq U .
\end{aligned}
$$

Let us now define a closed network with parameters (M', N', $\left.\mathrm{P}^{\prime}, \mathrm{B}_{\mathrm{i}}{ }^{\prime}, \mathrm{\square}_{\mathrm{i}}{ }^{\prime}, 0 \leq \mathrm{i} \leq \mathrm{M}\right)$. In order to define the closed analogue to the open Jackson network, assume that $\mathrm{M}^{\prime}=\mathrm{M}+1, \mathrm{~N}^{\prime}=\mathrm{U}, \mathrm{B}_{\mathrm{i}}{ }^{\prime}=\mathrm{B}_{\mathrm{i}}, \square_{\mathrm{i}}{ }^{\prime}=\square_{\mathrm{i}}, 1 \leq \mathrm{i} \leq \mathrm{M}, \mathrm{B}_{0}{ }^{\prime}=\mathrm{U}-\mathrm{L}, \mathrm{P}^{\prime}=\mathrm{P}$ is the $\mathrm{M}^{\prime} \mathrm{M}^{\prime}$ routing matrix, and each node $\mathrm{i}, 1 \leq \mathrm{i} \leq \mathrm{M}$, has the same service discipline (FCFS) and service time distribution (exponential) as the open network. Node 0 represents the external population of the open network, and it can be defined as a service center with FCFS discipline and load dependent service rate $\square_{0}\left(\mathrm{n}_{0}\right)=\mathrm{a}(\mathrm{n}), 0 \leq \mathrm{n}_{0} \leq \mathrm{B}_{0}{ }^{\prime}=\mathrm{U}-\mathrm{L}$, where $\mathrm{n}_{0}=\mathrm{U}-\mathrm{n}$, and $\mathrm{n}$ is the total population of the subnetwork $\{1,2, \ldots, M\}: n=\square_{1 \leq i \leq M} n_{i}, L \leq n \leq U$.

If node 0 works under BBS-O blocking and if the node 0 blocking function is defined as follows : $b_{0}\left(n_{0}\right)=d(n)$, where $n_{0}=U-n, L \leq n \leq U$, then, from the blocking type definition, one can immediately derive that the closed network behaves like the open Jackson network under STOP mechanism. Therefore, it is the analogue closed network of the open one. Fig. 4 shows the open Jackson network and its closed analogue.

The following theorem states that there exists an equivalence property between STOP and BBS-O blocking mechanisms. From this result it is possible to derive a new product form solution for a BBS-0 blocking network, as given by the following corollary. Note that, to our knowledge, this is the only product form network with BBS-O blocking type. 


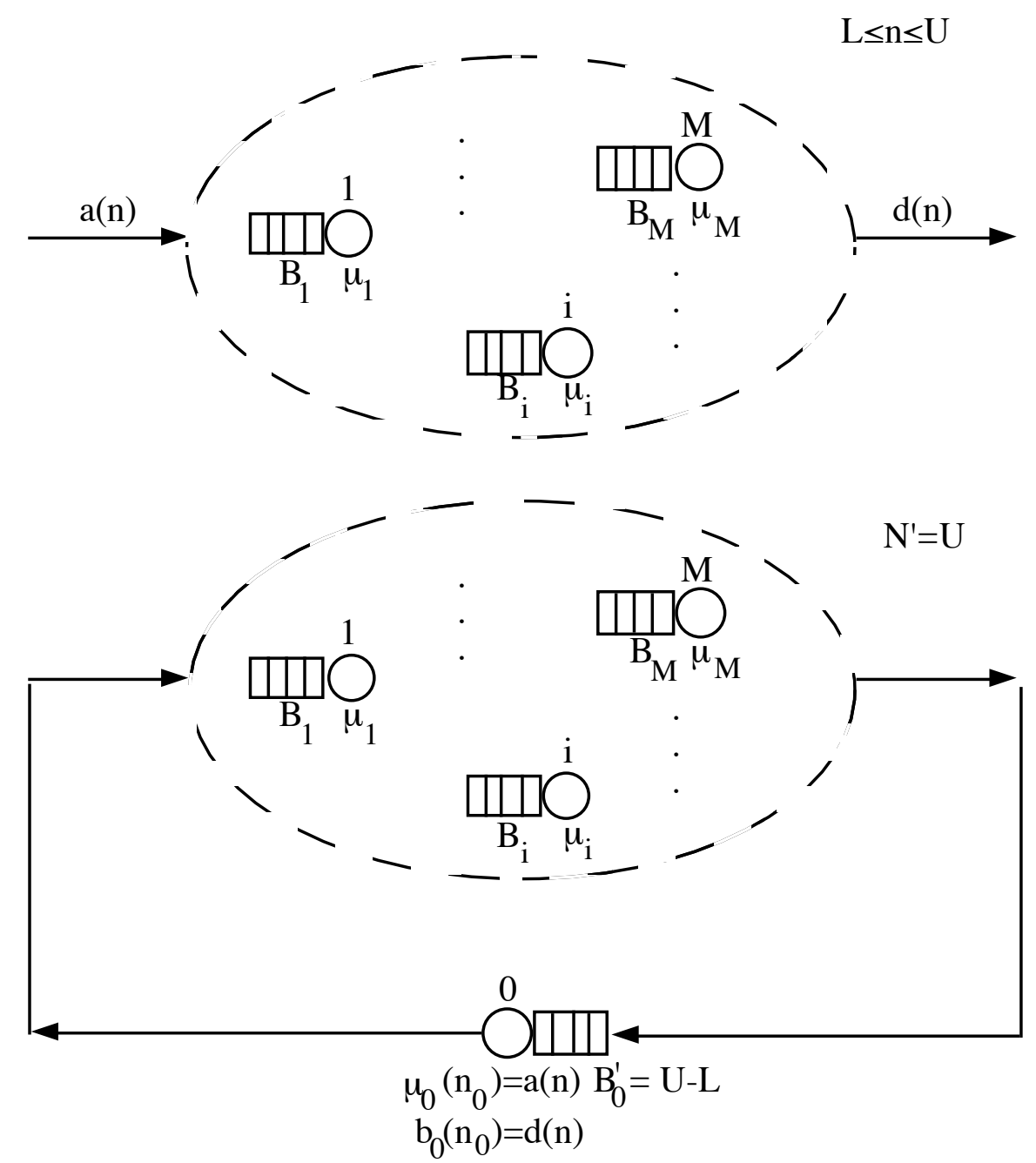

- Fig. 4 - An open Jackson network and its analogue closed network -

\section{Theorem EP9}

A Jackson network with $M$ nodes, under STOP blocking and with the total network population which satisfies $L \leq n \leq U, n=\sum_{i=1}^{M} n_{i}$, is reducible to its analogue closed network with $M+1$ nodes where node 0 works under BBS-O blocking with: $B_{0}=U-L$, $\square_{0}\left(n_{0}\right)=a(n), b_{0}\left(n_{0}\right)=d(n), n_{0}=U-n, 0 \leq n_{0} \leq B_{0}$. The bijective function between the two joint queue length distributions $\square^{S T O P}$ and $\square^{B B S-O}$ is given by :

$$
\operatorname{CPTOP}\left(n_{1}, n_{2} \ldots n_{M}\right)=\square^{B B S-O}\left(n_{0}, n_{1}, n_{2} \ldots n_{M}\right) \quad \square\left(n_{1}, n_{2} \ldots n_{M}\right), n_{0}=U-n .
$$

\section{Corollary 8}

A closed network analogue to an open Jackson network shows the following product form solution under BBS-O: 


$$
\square\left(\mathrm{n}_{0}, \mathrm{n}_{1}, \ldots, \mathrm{n}_{\mathrm{M}}\right)=\frac{1}{\mathrm{G}} \mathrm{V}(\mathrm{n}) \square_{\mathrm{i}=1}^{\mathrm{M}} \mathrm{g}_{\mathrm{i}}\left(\mathrm{n}_{\mathrm{i}}\right) \quad \square\left(n_{1}, n_{2} \ldots n_{M}\right), n_{0}=U-n .
$$

where

$$
\mathrm{V}(\mathrm{n})=\square_{\mathrm{l}=\mathrm{L}}^{\mathrm{n}-1} \mathrm{a}(\mathrm{l}) \quad \square \mathrm{n} \geq 0
$$

with $\quad \mathrm{a}(\mathrm{l})>0 \quad \mathrm{~L} \leq \mathrm{l}<\mathrm{U}, \quad \mathrm{a}(\mathrm{U})=0$

$$
\mathrm{g}\left(\mathrm{n}_{\mathrm{i}}\right)=\left(\mathrm{x}_{\mathrm{i}} / \mu_{\mathrm{i}}\right)^{\mathrm{n}_{\mathrm{i}}} \quad \square \mathrm{n}_{\mathrm{i}} \geq 0,1 \leq \mathrm{i} \leq \mathrm{M}
$$

Note that the product form solution (pf9) can be derived by applying formula (pf8) to the single class network, where nodes have FCFS service discipline.

A different case of queueing networks with arbitrary topology and a set of population constraints for subnetworks (or clusters) has been analyzed [39]. Van Dijk considers a closed single-class FCFS-exponential queueing network whose nodes are partitioned into $\mathrm{Z}$ disjoint subnetworks denoted by $\mathrm{C}_{\mathrm{W}}, 1 \leq \mathrm{W} \leq \mathrm{Z}$. Then we consider these population constraints in each subnetwork $\mathrm{W}$, i.e., the total subnetwork population, $\mathrm{n}_{\mathrm{W}}=$ $\square_{\mathrm{i} \square \mathrm{C}_{\mathrm{W}}} \mathrm{n}_{\mathrm{i}}$, is assumed to satisfy the constraint $\mathrm{n}_{\mathrm{W}} \leq \mathrm{B}_{\mathrm{W}}, 1 \leq \mathrm{W} \leq \mathrm{Z}$. In other words, $\mathrm{B}_{\mathrm{W}}$ is the maximum population admitted to subnetwork $\mathrm{W}$. We assume that the routing probabilities between nodes of the networks are defined as follows [39] :

$$
p_{i j}=\left\{\begin{array}{lll}
p_{i j}^{W} & i, j \square C_{W} & 1 \leq W \leq Z \\
p_{i 0}^{W} R_{W W^{\prime}} p_{0 j}^{W} & i \square C_{W}, j \square C_{W}, \text { with } W \neq W^{\prime}, 1 \leq W, W^{\prime} \leq Z
\end{array}\right.
$$

where $\mathrm{p}_{\mathrm{ij}}^{\mathrm{W}}$ are arbitrary probabilities within subnetwork $\mathrm{W}, \mathrm{i}, \mathrm{j} \square \mathrm{C}_{\mathrm{W}}, \mathrm{p}_{\mathrm{i} 0}^{\mathrm{W}}=\square_{\mathrm{j} \square \mathrm{C}_{\mathrm{W}}} \mathrm{p}_{\mathrm{ij}}^{\mathrm{W}}$ is the departure probability from node $\mathrm{i} \square \mathrm{C}_{\mathrm{W}}$ to a different subnetwork, and $\mathrm{p}_{\mathrm{i} 0}^{\mathrm{W}}$ is the probability that a job arriving at subnetwork $\mathrm{C}_{\mathrm{W}}$ enters node $\mathrm{i}, 1 \leq \mathrm{i} \leq \mathrm{M}, 1 \leq \mathrm{W} \leq \mathrm{Z}$. In other words, the routing among subnetworks is node independent, whereas upon job arrival at and within a subnetwork, an arbitrary routing is allowed.

The author defines an 'overall' STOP or RECIRCULATE blocking, that is, as long as one of the subnetworks is saturated, i.e., when for some $\mathrm{W} \mathrm{n}_{\mathrm{W}} \leq \mathrm{B}_{\mathrm{W}}, 1 \leq \mathrm{W} \leq \mathrm{Z}$, the blocking mechanism acts on all the nodes outside that subnetwork, that is, when the service at each node $\mathrm{i} \square \mathrm{C}_{\mathrm{W}^{\prime}}$, with $\mathrm{W}^{\prime} \neq \mathrm{W}, 1 \leq \mathrm{W}^{\prime} \leq \mathrm{Z}$, is stopped or recirculated. Both a product form solution and an equivalence property between STOP and RECIRCULATE blocking have been proved [39], as reported in the following theorems. 


\section{Theorem PF10}

A homogeneous closed Jackson network with single job class and a set of finite constraints defined on subnetworks shows the following product form solution under 'overall' STOP and 'overall' RECIRCULATE blocking mechanisms:

product form (4) with

$$
\begin{aligned}
& \mathrm{V}(\mathrm{n})=1 \quad \square \mathrm{n} \geq 0 \\
& \mathrm{~g}\left(\mathrm{n}_{\mathrm{i}}\right)=\left(\mathrm{x}_{\mathrm{i}} / \mu_{\mathrm{i}}\right)^{\mathrm{n}_{\mathrm{i}}} \quad \square \mathrm{n}_{\mathrm{i}} \geq 0,1 \leq \mathrm{i} \leq \mathrm{M}
\end{aligned}
$$

\section{Theorem EP10}

In a closed Jackson network with finite constraints defined on subnetworks and a single class of jobs, 'overall' STOP and 'overall' RECIRCULATE blocking mechanisms are identical.

\section{Conclusions}

Queueing networks with blocking and product form solution have been presented and compared, by considering different blocking mechanisms introduced in the literature. Blocking type definitions arise from modeling systems behaviors in many different fields including production, communication, and computer systems.

Equivalence properties between blocking types for product form networks have been presented and classified according to network topology, both in terms of joint queue length distribution and in terms of mean performance indices. Moreover, by combining product form results and equivalence properties, product form solutions have been extended to non-homogeneous networks where different nodes can have different types of blocking. This class of networks can be used to model complex systems, e.g., integrated computer-communication systems.

Finally, an equivalence relation between open Jackson networks with STOP blocking and the closed analogues has been defined, from which a new product form case for BBS-0 blocking was derived. 


\section{References}

[1] I.F. Akyildiz, Exact product form solution for queueing networks with blocking, IEEE Trans. Computer, C-36-1 (1987) 122-125.

[2] I.F. Akyildiz and H. von Brand, Central Server Models with Multiple Job Classes, State Dependent Routing, and Rejection Blocking, IEEE Trans. on Softw. Eng., SE-15-10 (1989) 1305-1312.

[3] I.F. Akyildiz and H. von Brand, Exact solutions for open, closed and mixed queueing networks with rejection blocking, J. Theor. Computer Science, 64 (1989) 203-219.

[4] I.F. Akyildiz and N. van Dijk, Exact Solution for Networks of Parallel Queues with Finite Buffers, in: Proc. Performance '90, Eds. P.J.B. King, I. Mitrani and R.J. Pooley (North-Holland, 1990) 35-49.

[5] I.F. Akyildiz and H.G. Perros, Special Issue on Queueing Networks with Finite Capacity Queues, Performance Evaluation, vol. 10, n. 3 (1989).

[6] T. Altiok, S.S. Stidham, A note on Transfer Line with Unreliable Machines, Random Processing Times, and Finite Buffers, IIE Trans., vol.14, 4, (1982), 125127.

[7] T. Altiok and H.G. Perros, Approximate analysis of arbitrary configurations of queueing networks with blocking, Ann. Oper. Res. 9 (1987) 481-509

[8] S. Balsamo and V. de Nitto Personè, Closed queueing networks with finite capacities: blocking types, product-form solution and performance indices, Performance Evaluation, vol.12, n.4 (1991) 85-102.

[9] F. Baskett, K.M. Chandy, R.R.Muntz, G. Palacios, Open, closed, and mixed networks of queues with different classes of customers, J. of ACM, 22 (1985) 248-260.

[10] O. Boxma and A.G. Konheim, Approximate analysis of exponential queueing systems with blocking, Acta Info., 15 (1981) 19-66.

[11] P. Caseau and G. Pujolle, Throughput capacity of a sequence of transfer lines with blocking due to finite waiting room, IEEE Trans. on Softw. Eng. 5 (1979) 631-642.

[12] K.M. Chandy, A.J. Martin, A characterization of product-form queueing networks, J. ACM, vol.30, n.2 (1983) 286-299.

[13] K.M. Chandy, J.H.Howard and D. Towsley, Product form and local balance in queueing networks, J. ACM, vol.24, n.2 (1977) 250-263. 
[14] J.W. Cohen, The multiple phase service network with generalized processor sharing, Acta Informatica, vol.12 (1979) 245-284.

[15] Y. Dallery and D.D. Yao, Modelling a system of flexible manufacturing cells, in: Modeling and Design of Flexible Manufacturing Systems, Kusiak Ed. (NorthHolland, 1986) 289-300.

[16] S. Gershwin and U. Berman, Analysis of transfer lines consisting of two unreliable machines with random processing times and finite storage buffers, AIIE Trans., 13, 1 (1981) 2-11.

[17] W.J. Gordon and G.F. Newell, Cyclic queueing systems with restricted queues, Oper. Res., 15 (1976) 286-302.

[18] F.S. Hillier and R.W. Boling, Finite queues in series with exponential or Erlang service times - a numerical approach, Oper.Res., 15 (1967) 286-303.

[19] A. Hordijk and N. van Dijk, Networks of queues with blocking, in: Performance '81, Ed. K.J. Kylstra (North Holland, 1981) 51-65.

[20] A. Hordijk and N. van Dijk, Networks of queues ; Part I: job-local-balance and the adjoint process; Part II : General routing and service characteristics, in: Lect. Notes in Control and Information Sciences, Eds. F.Baccelli and G.Fajolle (Springer-Verlag, 1983) 158-205.

[21] F.P. Kelly, Reversibility and Stochastic Networks, (Wiley, 1979).

[22] J.F.C. Kingman, Markovian population process, J. Appl. Prob., 6 (1969) 1-18.

[23] L. Kleinrock, Queuing Systems.Vol.1 :Theory, (Wiley,1975).

[24] A.G. Konhein and M. Reiser, A queueing model with finite waiting room and blocking, SIAM J.Comput, 7 (1978) 210-229.

[25] A.E. Krzesinski, Multiclass queueing networks with state-dependent routing, Performance Evaluation, vol.7, n.2 (1987) 125-145.

[26] J.R. Jackson, Jobshop-like queueing systems, Management Science, 10 (1963) 131-142.

[27] S.S. Lam, Queueing networks with capacity constraints, IBM J. Res. Develop. 21 (1977) 370-378.

[28] S.S. Lavenberg, Computer Performance Modeling Handbook, (Prentice Hall, 1983).

[29] M.F. Neuts, Two queues in series with a finite intermediate waiting room, J.Appl. Prob., 5 (1986) 123-142.

[30] R.O. Onvural, A Note on the Product Form Solutions of Multiclass Closed Queueing Networks with Blocking, Performance Evaluation, vol.10, n.3 (1989) 247-253. 
[31] R.O. Onvural, Survey of Closed Queueing Networks with Blocking, ACM Computing Surveys, vol. 22, n. 2 (1990) 83-121.

[32] R.O. Onvural and H.G. Perros, On equivalences of blocking mechanisms in queueing networks with blocking, Oper. Res. Letters (1986) 293-297.

[33] R.O. Onvural and H.G. Perros, Some equivalencies on closed exponential queueing networks with blocking, Performance Evaluation, vol.9 (1989) 111-118.

[34] H.G. Perros, Open queueing networks with blocking, in : Stochastic Analysis of Computer and Communications Systems, Ed. Takagi (North Holland, 1989).

[35] H.G. Perros, A bibliography of papers on queueing networks with finite capacity queues, Performance Evaluation, vol. 10, n.3 (1989) 225-260.

[36] B. Pittel, Closed exponential networks of queues with saturation: the Jackson-type stationary distribution and its asymptotic analysis, Math. Oper. Res. 4 (1979) 367-378.

[37] D.F. Towsley, Queueing network models with state-dependent routing, J. ACM 27 (1980) 323-337.

[38] N. van Dijk, On 'stop = repeat' servicing for non-exponential queueing networks with blocking, J. Appl. Prob., 28 (1991) 159-173.

[39] N. van Dijk, 'Stop = recirculate' for exponential product form queueing networks with departure blocking, Oper. Res. Lett., 10 (1991) 343-351.

[40] N. van Dijk and H.G. Tijms, Insensitivity in two node blocking models with applications, in: Proc. Teletraffic Analysis and Computer Performance Evaluation, Eds. Boxma, Cohen and Tijms (North Holland, 1986) 329-340.

[41] D.D. Yao and J.A. Buzacott, Modeling a class of state-dependent routing in flexible manufacturing systems, Ann. Oper. Res., 3 (1985) 153-167.

[42] D.D. Yao and J.A. Buzacott, Modeling a class of flexible manufacturing systems with reversible routing, Oper. Res., 35 (1987) 87-93.

[43] P. Whittle, Partial balance and insensitivity, J. Appl. Prob. 22 (1985) 168-175. 